\title{
Tuberculides Tuberculosas en población adulta de la Región Metropolitana, Chile. Serie de casos 2006-2010
}

\author{
Andrea Luna ${ }^{1}$, Martín Vidal$^{2}$, Zulema Torres ${ }^{3}$, Javier Arellano ${ }^{1,4}$.
}

\section{Resumen}

Introducción: Las tuberculides tuberculosas (TLTB) se subdividen en verdaderas de etiología atribuible al Mycobacterium tuberculosis y facultativas causadas por múltiples condiciones, entre ellas la tuberculosis. En Chile representan el 75\% de las tuberculosis cutáneas en la región metropolitana $(\mathrm{RM})$.

Objetivo: caracterizar las TLTB de la RM de acuerdo a epidemiología, clínica, estudio y manejo terapéutico.

Material y método: Estudio descriptivo retrospectivo del total de TLTB diagnosticadas por biopsia en los servicios de anatomía patológica de 4 hospitales públicos de la RM y un hospital privado de la RM en el quinquenio 2006-2010.

Resultados: 83 casos de TLTB, 59\% aportados por el sistema público, $84 \%$ de los pacientes fueron mujeres, $18 \%$ fueron tuberculides verdaderas y $82 \%$ tuberculides facultativas. A pesar de ser el tratamiento antituberculoso la elección en las tuberculides verdaderas, sólo se indicó en el $65 \%$ de los casos y de estos, $45 \%$ realmente lo realizó. Sólo el 4,5\% de las tuberculides facultativas de esta serie tuvieron indicación de tratamiento antituberculoso, realizándose en el 100\% de los casos.

Conclusión: Existe seguimiento insuficiente a través del tiempo en estos pacientes, dificultando dimensionar el éxito real de los tratamientos indicados. Se evidencia una falla en la coordinación al momento de derivar los pacientes al programa de control de la tuberculosis.

Palabras clave: Tuberculosis cutánea; Infecciones bacterianas de la piel Dermatología Epidemiología de tuberculosis cutánea.

\section{SUMMARY}

Introduction: Tuberculids are subdivided in true tuberculids, which are associated to mycobacterium tuberculosis, and facultative tuberculids which can be caused by several conditions, including tuberculosis. In Chile tuberculids represent $75 \%$ of cutaneous tuberculosis in the metropolitan region (MR).

Objective: to characterize tuberculids according to epidemiology, clinical, study and therapeutic management.

Methods: Descriptive study of the total tuberculids diagnosed by biopsy in the pathology services of 4 public hospitals of the MRI and a private hospital of the MRI in the five-year period 20062010.

Results: 83 cases of tuberculids, $59 \%$ where provided by the public system, $84 \%$ of the patients were women, $18 \%$ were true tuberculids and $82 \%$ facultative tuberculids. Despite being antitubercular therapy the first line treatment for true tuberculids, this treatment was prescribed in $65 \%$ of cases and only $45 \%$ of these were actually treated. Only $4.5 \%$ of the facultative tuberculids in this series had indication of antitubercular therapy, performed in $100 \%$ of cases.

Conclusions: There is insufficient follow up in these patients, making it difficult to assess the real success of the treatment. Is evident the lack of coordination when referring patients to the tuberculosis control program.

Key words: cutaneous tuberculosis; bacterial skin diseases; Dermatology; tuberculosis, cutaneous/epidemiology.

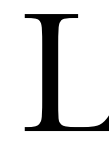
a tuberculosis (TB) es hoy una de las enfermedades reemergentes más importantes de nuestra era. Chile se encuentra en una situación privilegiada, con una tasa de incidencia de 16/100.000 habitantes, y es uno de los 5 países de baja prevalencia en la región junto a Cuba, Uruguay, Puerto Rico y Costa Rica. ${ }^{1-4}$ La TB cutánea representa entre un $1 \%$ y $4,8 \%$ del total de las TB extra pulmonares y cursa con otras formas de TB entre un $28 \%$ y $70 \%$ de los casos. ${ }^{5-10}$ Se subdivide en TB cutáneas verdaderas y tuberculides tuberculosas (TLTB),

'Departamento de dermatología, Hospital San Borja Arriarán, Universidad de Chile. Santiago, Chile. ${ }^{2}$ Facultad de medicina, Universidad Finis Terrae. Santiago, Chile. ${ }^{3}$ Programa Nacional de Control de la Tuberculosis, Ministerio de Salud. Santiago, Chile. ${ }^{4}$ Departamento de Dermatología, Sede centro, Universidad de Chile. Santiago, Chile.

Correspondencia: Andrea Luna Heine. Correo electrónico: amlh2009@gmail.com, Teléfono: +56966594356 Dirección: Avenida Santa Rosa 1234, Santiago, Chile. Código Postal: 8360160. 
las que a su vez son clasificadas en tuberculides verdaderas de etiología atribuible al Mycobacterium Tuberculosis y tuberculides facultativas, causadas por múltiples agentes y condiciones, entre ellas la TB (Tabla 1).

Tabla 1. Clasificación de las distintas formas clínicas de TLTB10

\begin{tabular}{l|l}
\hline Tuberculides verdaderas & Tuberculides facultativas \\
\hline Eritema indurado de Bazin & Vasculitis nodular \\
Liquen escrofuloso & Eritema nodoso \\
Tuberculide papulonecróticas & \\
Flebitis granulomatosa nodular & \\
\hline
\end{tabular}

TLTB: Tuberculide Tuberculosa

Las TLTB verdaderas y las facultativas en donde se confirme su etiología tuberculosa deben ser notificadas y tratadas con antifimicos por ser TB extra pulmonares. ${ }^{5,9-14}$

En Chile, las TLTB representaron el $75 \%$ de las Tuberculosis Cutáneas en la Región Metropolitana durante el quinquenio 2006$2010,{ }^{15}$ por lo que como objetivo general en este estudio, se pretende caracterizar las TLTB de acuerdo a epidemiología, clínica, estudio y manejo terapéutico en cinco hospitales de la Región Metropolitana. Con el objetivo específico de conocer los exámenes más frecuentemente solicitados para apoyar o descartar etiología tuberculosa en TLTB y verificar cumplimiento de indicación de tratamiento antituberculoso.

\section{Materiales y MÉTOdoS}

Estudio descriptivo retrospectivo, basado en una serie de 83 casos de TLTB, diagnosticadas durante el quinquenio 2006-2010 por biopsia y clínica en los servicios de anatomía patológica de cuatro hospitales públicos de la región metropolitana (Hospital San José, Hospital Barros Luco Trudeau, Hospital Salvador y Hospital San
Borja Arriarán) que representan el 56\% de la población adulta usuaria del sistema público de Salud (cobertura de 2.655.350 usuarios inscritos en FONASA), y un Hospital privado de la Región Metropolitana (Hospital Clínico Universidad de Chile) correspondiente al segmento socio-económico predominante C2-C33.

Se revisaron las bases de datos de los servicios de anatomía patológica de estos hospitales, seleccionando a aquellos casos en los al menos una biopsia fue informada por un dermatopatólogo como sugerente o compatible con TLTB y que cumplieran con los siguientes criterios de inclusión:

- Adulto con diagnóstico sugerente, compatible o probable de TLTB en la histopatología y con ficha clínica disponible.

Y que no cumpliera con los criterios de exclusión:

- Menores de 15 años, otras TB que no cursen simul-

\section{PUNTOS CLAVE}

La TB cutánea es una forma poco frecuente de TB. Las TLTB son subtipos de TB cutánea con escasa infomación epidemiológica en Latinoamérica.

Se evidencia predominio de tuberculides facultativas y de afección femenina. Falta de registro dificulta determinar características clínicas. Tratamiento y seguimiento de esta patología es insuficiente.

Falencias constatadas deberían inducir a mejorar calidad de registro de datos, estrategias diagnósticas y seguimiento de estas entidades. táneamente con TLTB durante el periodo de interés.

Se realiza selección de TLTB mediante limpieza de datos, considerando como casos de tuberculides tuberculosa, las que cumplieran el criterio de diagnóstico dado por tres elementos: biopsia, clínica compatible y algún elemento epidemiológico de apoyo como antecedentes de contacto pasado o reciente con $\mathrm{TB}$, cursar de forma concomitante con TB en otro sitio o presentar derivado proteico purificado (PPD) positivo.

Cruce de datos con registros nacionales para enfermedades de notificación obligatorias (ENO) y Registro de tratamientos antituberculoso del Ministerio de Salud (MINSAL). Análisis de la base de datos realizado con softwares Excel y SPSS ${ }^{17}$ para variables con mejor integridad de registro de dato.

\section{Resultados}

De los 83 casos de TLTB encontrados, el 59\% fueron aportados por el sistema público y el $41 \%$ por el sistema 
privado. La distribución por género tuvo un marcado predominio femenino (84\%), con un promedio de edad de 42 años (mediana 41 años) y un rango de edad de 17 a 86 años.

El 18\% fueron tuberculides verdaderas y el $82 \%$ tuberculides facultativas. La falta de integridad del registro de datos no permitió calcular las formas clínicas de cada tipo de TLTB.

En relación a las características de las TLTB se encontró que la duración del episodio actual tenía un promedio de 2,4 meses (mediana 2; moda 3) y rango de 0,1 a 7 meses. La duración de la enfermedad tuvo un promedio de 3,4 años desde el primer episodio (mediana 3; moda 3) con un rango de una semana a 11 años. Respecto a la distribución de las lesiones, estas se ubicaron en extremidades inferiores con otro sitio anatómico adicional en el 31\%, exclusivamente en extremidades inferiores en el 19\% de los casos y sólo en extremidades superiores en el 4\%. Según localización de lesiones se encontró bilateralidad en un 55\% de los pacientes. La distribución anteroposterior en paniculitis versus histología no se pudo calcular por falta de integridad del registro de dato. El 58\% de los pacientes presentó paniculitis dolorosas, con predomino de este síntoma en el eritema nodoso, sin una diferencia estadísticamente significativa frente al eritema indurado de Bazin (X2 0,088), en correlato dolor-histología.

Los pacientes con TLTB presentaron con frecuencia variadas comorbilidades (Tabla 2).

Tabla 2. Comorbilidades en TLTB

\begin{tabular}{l|c|c}
\hline PATOLOGÍA & CASOS & PORCENTAJE \\
\hline Diabetes mellitus & 7 & 9 \\
Trastornos tiroideos & 7 & 9 \\
Mesenquimopatías & 6 & 7 \\
Embarazo y puerperio & 5 & 6 \\
DCA & 4 & 5 \\
Depresión severa & 3 & 2 \\
Asma & 3 & 2 \\
Anemia & 3 & 2 \\
Insuficiencia renal & 3 & 2 \\
VIH & 1 & 1 \\
Alcoholismo & 1 & 1 \\
Vitíligo & 1 & 1 \\
\hline
\end{tabular}

TLTB: Tuberculide tuberculosa

DCA: Dermatitis de contacto alérgica

VIH: Virus de inmunodeficiencia humana
Dentro de los exámenes solicitados para descartar otras patologías causales de la TLTB el examen pedido con mayor frecuencia fue el ASO/ASLO en el 52\% de los casos, en el 28\% de los casos solicitaron anticuerpos antinucleares (ANA), seguido por el VDRL solicitado en el $15 \%$ y TSH en el 12\%, también fueron solicitados complemento en el $8 \%$, serología para virus hepatitis B,C y/o VIH en el 8\%, anticuerpos anti citoplasma de neutrófilos (ANCA) en el 8\% y factor reumatoideo (FR) en el $7 \%$ (Tabla 3$)$.

Tabla 3. Exámenes solicitados para apoyar el diagnóstico de TB.

\begin{tabular}{l|c}
\hline Examen & $\begin{array}{c}\text { Porcentaje } \\
\text { de solicitud }\end{array}$ \\
\hline Biopsia & 100 \\
Hemograma con VHS & 72 \\
PPD & 49 \\
Radiografía de tórax o TAC & 42 \\
Baciloscopía y/o cultivo de piel & 16 \\
Baciloscopía y/o cultivo de expectoración & 6 \\
Baciloscopía y/o cultivo de orina & 5 \\
PCR para TB & 4 \\
ADA & 1 \\
IGRAs & 1 \\
\hline
\end{tabular}

VHS: velocidad de sedimentación globular

PPD: derivado proteico purificado

TAC: tomografía axial computada

PCR: reacción en cadena de polimerasa

TB: tuberculosis

ADA: adenosin deaminasa

IGRAs: ensayo de liberación de interferón gamma

Los tratamientos indicados y su adherencia fueron variables en TLTB. En un 10\% del total de TLTB se indicó antibióticos antifimicos, indicación que abarcó al 65\% de las TLTB verdaderas. Otras medidas farmacológicas fueron utilizadas para alivio de síntomas y cobertura de otros microorganismos (Tabla 4).

\section{Discusión}

Al ser la TB cutánea una patología de resolución en el nivel secundario, los casos obtenidos deben representar un número bastante aproximado a la realidad.

La mayor parte de los pacientes presentados en esta serie 
46 - Andrea Luna

Tabla 4. Principales tratamientos indicados en TLTB.

\begin{tabular}{|c|c|c|}
\hline tratamiento realizado & Porcentaje & Observaciones \\
\hline Yoduro de potasio & $18 \%$ & $\begin{array}{l}\text { Dosis usada } 300 \text { mg cada } 8 \text { horas tiempo variable, } \\
\text { elección más frecuente en eritema nodoso. }\end{array}$ \\
\hline AINES & $18 \%$ & $\begin{array}{l}\text { Fármacos más frecuentes: naproxeno, ketoprofeno, indometacina } \\
\text { y diclofenaco. En dosis habituales. Segunda elección en frecuencia } \\
\text { en eritema indurado de Bazin y eritema nodoso. }\end{array}$ \\
\hline Antibióticos no antifímicos & $13 \%$ & Penicilina benzatina 1,2 millones IM, 1 o más dosis, cefalosporinas y sulfas. \\
\hline Corticoides orales y/o tópicos & $11 \%$ & $\begin{array}{c}\text { Prednisona hasta } 0,5 \mathrm{mg} \mathrm{mg} / \mathrm{kg} / \mathrm{dí} \text { en cura corta y dosis decreciente, } \\
\text { clobetasol } 0,05 \% \text { tópico durante tiempo variable. }\end{array}$ \\
\hline Antibióticos antifímicos & $10 \%$ & $\begin{array}{l}10 \% \text { del total de tuberculides. } \\
\text { Antifímicos en TLTB verdaderas, indicación } 65 \% \text { de los casos, } \\
\text { sólo el } 45 \% \text { efectuó tratamiento. } \\
\text { Antifímicos en TLTB facultativas, indicadas sólo en } 3 \text { casos; } \\
\text { el } 100 \% \text { efectuó tratamiento. }\end{array}$ \\
\hline $\begin{array}{l}\text { Medidas generales sin } \\
\text { tratamiento asociado }\end{array}$ & $4 \%$ & Reposo indicado solo en eritema nodoso. \\
\hline
\end{tabular}

AINES: Antiinflamatorio no esteroideo

TLTB: Tuberculide tuberculosa

IM: Intramuscular

provienen del servicio público de Salud, pero llama la atención que el único hospital privado del estudio aportó el $41 \%$ de los casos. No hay otros estudios nacionales que permitan comparar este hallazgo.

El amplio predominio del género femenino en este estudio y el promedio de edad, relativamente bajo, son concordantes con lo descrito en otros trabajos. ${ }^{10}$

Las TLTB facultativas fueron más frecuentes que las TLTB verdaderas. Si bien el eritema indurado de Bazin (una forma de TLTB verdadera) es la forma más frecuente de TLTB descrita en la literatura, ${ }^{16-18}$ en nuestro estudio no fue posible determinar las diferentes formas clínicas de TLTB debido a la falta de integridad del registro de datos.

La distribución de las lesiones tuvo predominio en extremidades inferiores, lo que coincide con otros estudios. ${ }^{10}$

La TB se asocia a factores de riesgo como infección por VIH, diabetes mellitus, enfermedad renal crónica, cáncer y otras formas de inmunosupresión. ${ }^{19}$ Lo mismo ocurre con las TB cutáneas, lo que se manifestó en las comorbilidades presentadas en los pacientes de este estudio. ${ }^{20}$

Las mejores herramientas diagnósticas de TB cutánea descritas son el estudio histológico, el cultivo de piel y la PCR de ADN micobacterial. Estas dos últimas técnicas se consideran criterio absoluto de TB cutánea. Sin embargo, las TLTB pueden no presentar bacilos ácidoalcohol resistente, por lo que la PCR y cultivo podrían ser menos útiles. ${ }^{3} \mathrm{El}$ estudio histológico fue realizado en el $100 \%$ de los pacientes de este estudio, lo que indica que el criterio de inclusión utilizado en la selección de casos fue bien escogido. Sin embargo, sólo en el 4\% de los casos se realizó PCR de TB y sólo en el 16\% cultivo de piel. La baja indicación de estos estudios se podría deber a su elevado costo en el caso de la PCR y a la demora relativa en detectar crecimiento bacteriano $(2$ a 8 semanas) en el caso del cultivo. ${ }^{3}$ Estudios como PPD y radiografía o TAC de tórax, realizados en el 49\% y 42\%, respectivamente, son mandatorios para el estudio de posible TB interna concomitante, especialmente pulmonar.

El principal pilar terapéutico consiste en administrar antibióticos antifímicos, siendo una terapia muy efectiva en las TLTB. ${ }^{3,11,12,20-23}$ Sin embargo, este manejo fue indicado en el $10 \%$ de los pacientes de este estudio, abarcando sólo al $65 \%$ de los pacientes con TLTB verdadera. Si bien esta cifra ya es baja, el cumplimiento del tratamiento fue completado sólo en el $45 \%$, porcentaje que dista de otros estudios, donde la adherencia al tratamiento es 
de hasta un $94,5 \% .{ }^{10}$ Esta diferencia puede deberse a una falla de coordinación eficiente al momento de derivación de los pacientes que deben ingresar al programa de control de tuberculosis. Medidas de soporte, yoduro de potasio y tetraciclinas fueron utilizados en algunos pacientes. Estos y otros como dapsona, sales de oro, colchicina y antimaláricos se describen como tratamientos adyuvantes y algunos estudios han reportado alivio de síntomas e inducción de remisión, sin embargo, no han podido evitar recurrencia tardía. ${ }^{21,24}$ Los corticoides, también utilizados en algunos pacientes de este estudio, tienen por finalidad generar desensibilización y disminuir reacciones de hipersensibilidad. ${ }^{21,24}$ El manejo y seguimiento insuficiente de estas entidades disminuye la cura, aumenta la recidiva y resistencia antibiótica. ${ }^{23}$ Este estudio evidencia falencias en la derivación al sistema público y en el seguimiento en el tiempo, que impiden iniciar y comprobar la efectividad del tratamiento.

\section{Conclusiones}

Las TLTB son un desafío diagnóstico. Si bien en este estudio fue más frecuente la afectación del género femenino y la forma predominante de TLTB fue la facultativa, la mala calidad del registro de datos en fichas clínicas limitó el aporte de conocimientos que podría haber generado este estudio. Se hace necesario un estudio exhaustivo para descartar otras patologías en TLTB facultativas y para apoyar o descartar la causa tuberculosa. Este estudio evidencia una falla de coordinación eficiente al momento de derivar a los pacientes que deben ingresar al programa de control de TB. Existe un seguimiento insuficiente de los pacientes con TLTB en el tiempo, lo que dificulta dimensionar el éxito real de los tratamientos indicados.

\section{REFERENCIAS}

1. Zuñiga M, Rojas M. Programa Nacional de Control de la Tuberculosis. Año 2000: Avances hacia la eliminación. Rev Chil Enferm Respir. 2002;18:55-63

2. Zúñiga M. Chile inicia la primera etapa de la eliminación de la tuberculosis. Rev Chil Enferm Respir. 2000;16:105-10

3. MINSAL. Programa Nacional de Control de la Tuberculosis. Manual de Organización y Normas Técnicas 2005. Disponible en: www.minsal.gob.cl/portal/url/item/80304817 lacc60f8e0400101 lf 0148e2.pdf [Consultado el 20/12/2015]
4. World Health Organization. Tuberculosis (TB). Disponible en: www. who.int/tb [Consultado el 22/12/2015]

5. Almaguer J, OcampoJ, Rendón A. Panorama actual en el diagnóstico de la tuberculosis. Actas Dermosifiliogr. 2009;100:562-70

6. Pérez L, Muñoz C, Benavides A, González S. Eritema Indurado de Bazin. Dermatol Pediatr Lat. 2005;3:142-8

7. Semaan R, Traboulsi R, Kanj S. Primary Mycobacterium Tuberculosis complex cutaneous infection: report of two cases and literature review. Int J Infect Dis. 2008;12:472-7

8. Concha M, Fich F, Rabagliati R, Pinto C, Rubio R, Navea O, et al. Tuberculosis Cutánea: Reporte de dos casos y revisión de la literatura. Rev Chil Infectol. 2011;28:262-8

9. Tincopa O, Jave B. Tuberculosis cutánea estudio clínico, histopatológico y de frecuencia. Dermatol Perú. 1999;9:10-8

10. García J, Monteagudo B, Mariño A. Tuberculosis cutánea estudio descriptivo de 15 años. Enferm Infecc Microbiol Clin. 2008;26:205-11

11. Monteagudo B, García J, León E, Mariño A. Incidencia de la Tuberculosis cutánea en pacientes con otro tipo de tuberculosis. Piel. 2007;22:267-70

12. Bravo F, Gotuzzo E. Cutaneous Tuberculosis. Clin Dermatol. 2007;25:173-80

13. Kumakawa Z, Santos R, Alvarez P, Angulo N, Meza B, León A. Nuevo espectro de tuberculide papulosa: aspectos clínicos e histopatológicos a propósito de un caso. Folia Dermatol Perú. 2009;21:25-9

14. Bravo F. Tuberculides en el siglo XXI. Folia Dermatol Perú. 2009;20:61-2

15. Luna A. Enfoque diagnóstico en Tuberculosis Cutánea. Unplubished. [Tesis de Dermatología]. Santiago: Universidad de Chile; 2012

16. Tigoulet F, Fournier V, Caumes E. Clinical forms of the cutaneous tuberculosis. Bull Soc Pathol Exot. 2003;96:362-7

17. Tincopa O, Sanchez L. Tuberculosis cutánea. Dermatol Peru. 2003;13:195-217

18. Hamada M, Urabe K, Moroi Y, Miyazaki M, Furue M. Epidemiology of cutaneous tuberculosis in Japan: a retrospective study from 1906 to 2002. Int J Dermatol. 2004;43:727-31

19. Ramírez M, Menéndez A, Noguerado A. Extrapulmonary tuberculosis: an overview. Rev Esp Sanid Penit. 2015;17:3-11

20. Frankel A, Penrose C, Emer J. Cutaneous tuberculosis. J Clin Aesthetic Dermatol. 2009;2:19-27

21. Gavazzoni M, Quaresma M, da Costa J, Filho F, do Nascimento L, Azulay D. Update on cutaneous tuberculosis. An Bras Dermatol. 2014;89:925-38

22. Belo dos Santos J, de Oliveira M, Figueiredo A, Gomes da Silva P, Ferraz C, Silveira de Medeiros V. Cutaneous tuberculosis: epidemiologic, etiopathogenic and clinical aspects - Part I. An Bras Dermatol. 2014;89:219-28

23. Belo dos Santos J, de Oliveira M, Figueiredo A, Gomes da Silva P, Ferraz C, Silveira de Medeiros V. Cutaneous tuberculosis: diagnosis, histopathologyand treatment - Part II. An Bras Dermatol. 2014;89:545-55

24. Kakkanatt A, Krishnan P, Divakaran A, Erythema induratum of Bazin - Tuberculosis in disguise. Journal of Dermatology \& Dermatologic Surgery. 2015;19:66-8 